\title{
THE IMMUNOCHEMICAL BASIS FOR PENICILLIN ALLERGY
}

\author{
Charles W. Parker \\ From the Division of Allergy and Immunology, Department of Medicine, \\ Washington University School of Medicine, St. Louis, Missouri 63110.
}

Although the incidence of drug allergy in general is low, there are a few drugs which are notable exceptions. For example, Nirvanol, a drug which was once used for treatment of chorea, caused allergic symptoms in nearly every patient (Sherman, 1947). Moreover, among organic molecules which are not of therapeutic value but which are analogous to drugs structurally, many examples can be cited where there is a high sensitizing capacity (Landsteiner, 1945). These chemicals which are potent sensitizers have the common property that they are able to react with proteins to form a stable bond. The two main types of combination between drugs and proteins are shown in Fig. 1. After the adsorption of a drug to protein, the complex can be readily dissociated and both drug and protein are recovered in their original form. Nearly all drugs are bound reversibly to some degree by protein, especially the serum albumins. In forming a stable or covalent bond a portion of the drug molecule must be in a reactive form. Reaction takes place with amino acid residues which are capable of assuming a charge on their side chains, such as lysine, tyrosine and histidine. The stability of the bond formed will in many instances be comparable to that of a peptide bond.

Because potent sensitizers do form stable bonds with protein in vitro, it has been postulated that immunization to a simple chemical requires the combination of that chemical with body protein; in other words, that a covalent bond is formed in vivo (Landsteiner, 1945; Eisen, 1959). In accord with this idea is the fact that a covalent conjugate, formed by reacting a protein and a chemical in vitro, is a potent antigen. By contrast, the reversible combination of a chemical with a protein does not appear to confer immunogenicity (Eisen, 1959).

The application of this general concept to

Supported by Grants of the United States, Public Health Service (A1-04646-02) and $5 K 3$ (A1-9881-02).
1. Adsorption of a drug to protein

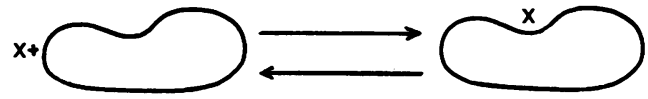

2. Covalent binding of a drug to protein

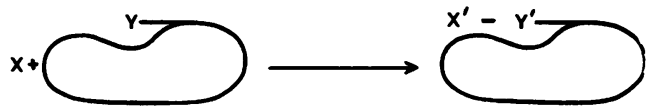

Fig. 1.-Types of binding between drugs and proteins. $X$ is any drug.

$Y$ is a functional group of an amino acid $\bar{\sigma}$ residue of a protein.

$\mathrm{X}^{\prime}-\mathrm{Y}^{\prime}$ is the product formed when $\mathrm{X}$ combines covalently with $\mathbf{Y}$.

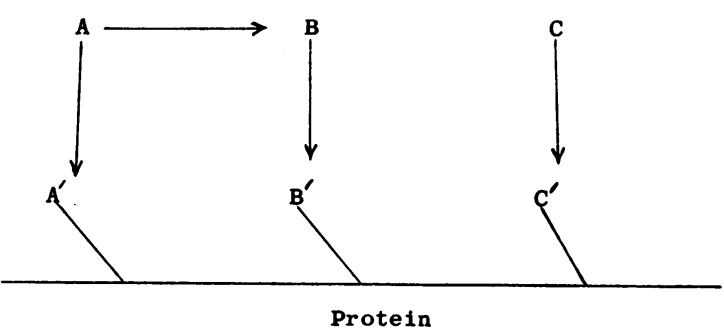

FIG. 2.-The formation of antigen.

drug allergy is not as straightforward as it might seem. It is seldom possible to demonstrate that a given drug, $\mathrm{A}$, which causes allergy, reacts directly with a protein to produce a covalently bound protein substituent, $A^{\prime}$ (Fig. 2). This does not invalidate the concept, however. As sources of antigen, we must consider not only drug A itself but also its breakdown products, $B$ and $C$, contaminants of $\mathrm{A}$. If $\mathrm{B}$ and $\mathrm{C}$ have a high degree of protein reactivity, microgram amounts may 
TABLE I

Representative Hemagglutination Inhibition Patterns

\begin{tabular}{lllrrrrr}
\hline & & & \multicolumn{5}{c}{ Serum } \\
& & & 1 & 2 & 3 & 4 & 5 \\
Penicilloyl & $\ldots$ & $\ldots$ & 1 & 7 & $>100$ & 15 & $>10$ \\
Penicillenate & $\ldots$ & $\ldots$ & 30 & 1 & 5 & 50 & $>10$ \\
Penilloate & $\ldots$ & $\ldots$ & $>50$ & 4 & 1 & 10 & $>10$ \\
Penamaldate & $\ldots$ & $\ldots$ & 3 & 1 & 16 & 10 & 1 \\
Polypenicilloyl & $\ldots$ & $\ldots$ & 15 & $\overline{7}$ & $>100$ & 1 & $>10$ \\
Penicillin & $\ldots$ & $\ldots$ & 50 & 5 & 10 & 30 & $>10$ \\
Penicilloate & $\ldots$ & $\ldots$ & 5 & $->100$ & 15 & $>10$ \\
\hline
\end{tabular}

Hemagglutination inhibition patterns observed with sera from patients with penicillin allergy. Each vertical column represents an individual serum. In a given column the penicillin derivative designated by numeral 1 is the best inhibitor. Other numbers refer to concentrations of other derivatives which produce a corresponding degree of inhibition. For example, in column 1, penamaldate must be present in a three fold greater concentration than penicilloyl in order to produce equivalent inhibition.

be sufficient to produce an allergic response. Since many drugs are given therapeutically in gram amounts, we must consider degradation reactions and contaminants which represent only a very small fraction of the total drug given. Moreover, the very fact that an antigenic precursor has a high degree of chemical reactivity may preclude its isolation in the free state as an in vivo degradation product (Parker, 1964a).

Because of some of these difficulties, validation of the general concept that antibodies in drug allergy are to protein bound derivatives of a drug rather than the drug itself has only been accomplished in the past few years. The available evidence is at present limited to penicillin allergy. The evidence in penicillin allergy is now quite convincing; it includes the results of skin testing and hemagglutination in man as well as extensive animal experimentation (Parker, Shapiro, Kern and Eisen, 1962; Thiel, Mitchell and Parker, 1964; De Weck, 1962; Levine and Ovary, 1961; De Weck and Eisen, 1960; Levine, 1960). As an example of the type of data available, let us consider the results of hemagglutination inhibition. One can take sera or globulin fractions from patients with penicillin allergy and in the majority of instances demonstrate substantial hemagglutination titers with sensitized cells (Thiel et al., 1964; Ley, Harris, Brinkley, Liles, Jack and Cahan, 1958; Van Arsdel, Tobe and Pasnick, 1963; Harris and Vaughn, 1961; Reisman, Rose, Witebsky and Arbesman, 1962).
It is probable that the types of antibodies primarily involved in direct hemagglutinatio are not responsible for allergic symptoms. But hemagglutination does afford a means of evaluating antibody specificity. Columns 1of Table I represent results of hemagglutinatio inhibition with five individual globulin frac음 tions. In Column 1, the derivative represen $\frac{\bar{s}}{5}$ ted by the lowest number, penicilloyl, is the best inhibitor of hemagglutination. The number 50 by penicillin in column 1 indicates that penicillin must be present at a 50 fold higher concentration in order t $\omega_{\omega}$ produce a corresponding degree of inhibition? One can presume, therefore, that the antio bodies responsible for agglutination are specifie for penicilloyl, not penicillin, and that the weak inhibitory activity of penicillin is dues to cross reactivity. The hemagglutinationg inhibition pattern in column 1 is the most common one, being observed with most posi $\nexists$ tive globulin fractions; occasionally one canp find a globulin fraction where a penicillin derivative other than penicilloyl is the best inhibitor (Thiel et al., 1964). Examples of these are shown in columns $2,3,4,5$ wheres penicillenate, penilloate, penamaldate and polypenicilloyl are better inhibitors tha penicilloyl. One can also obtain hemagglutin tion inhibition patterns which indicate the antibodies of at least two specificities are present. In every instance one or more deris vatives of penicillin capable of existing in covalent linkage with protein has been a.़ better inhibitor than penicillin itself.

Based on these and other studies I wouldo suggest that pathways shown on Fig. 3 are involved in formation of antigen. Penicilloyl, the derivative shown in the lower right and left hand corners of Fig. 3 (IVa and IVb), appears to be the most important antigenicdeterminant in penicillin allergy. It differs 3 . from the original penicillin molecule in that the $\beta$-lactam ring has opened and there is a₹ stable attachment to an $\varepsilon$-amino group of ao protein lysyl residue. As indicated on Fig. 3, it can arise either by a direct coupling mechan-o ism or through the intermediary formation of penicillenic acid (III). My own view at presento is that probably both mechanisms contributen to formation of protein-bound penicilloyl 서 in vivo (Thiel et al., 1964).

In addition to penicillenic acid (III), peni-e cilloic acid (VII), penamaldate (IX), and 6-aminopenicillanic acid (I) can be presumed $\stackrel{?}{+}$ to be intermediates in the formation of anti- 

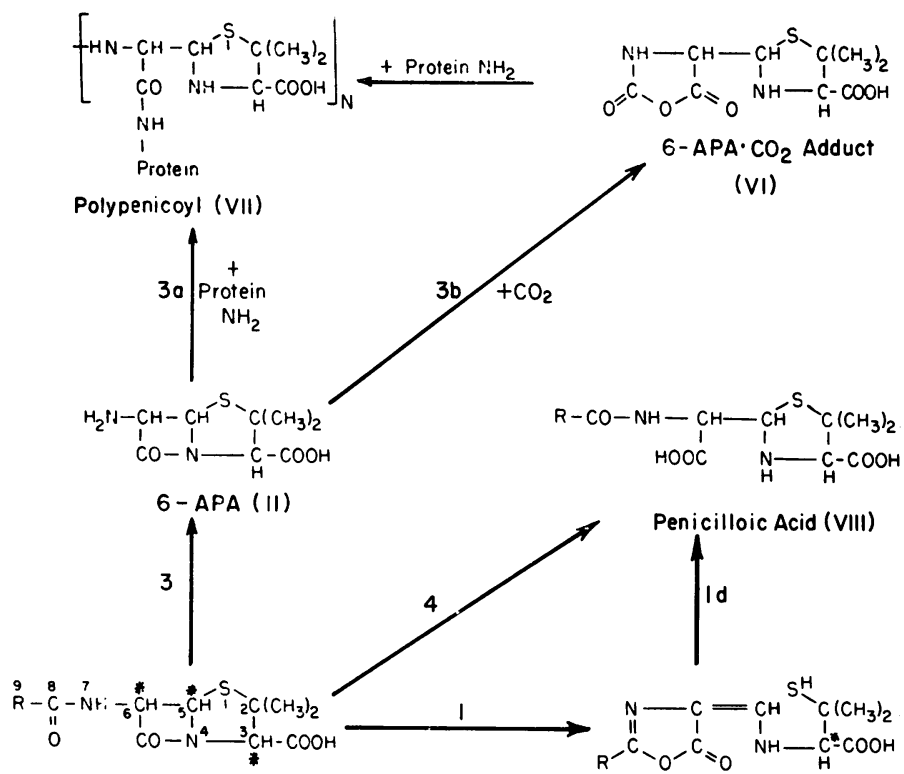

Penicillin (1)

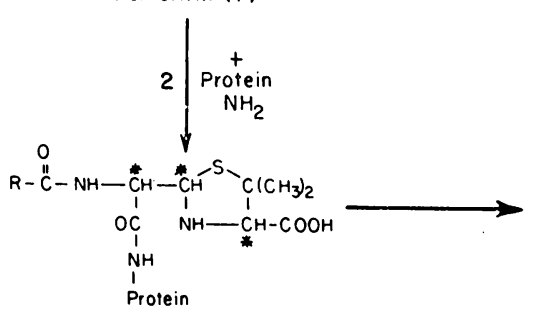

De Penicilloyl (IVa)
Penicillenic Acid (III)

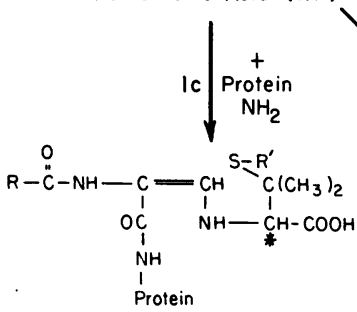

Penamaldoyl (V)

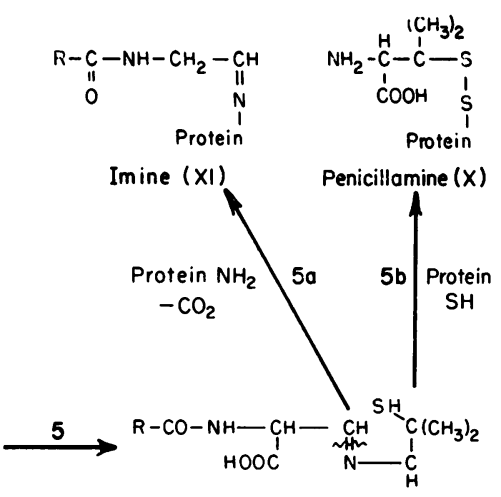

Penamaldate (IX)

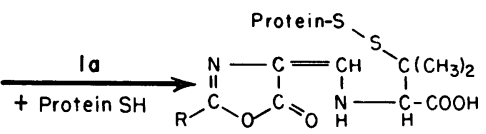

Penicillenate (XII)

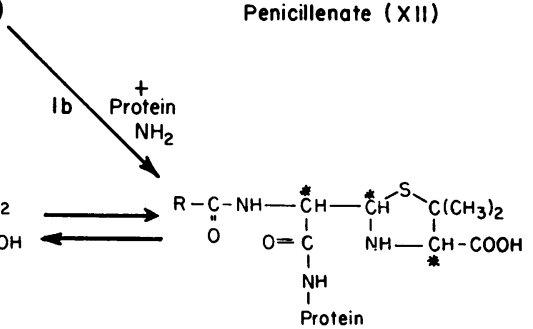

Mixture of Diastereoisomers of Penicilloyl (IVb)

FIG. 3.-Proposed pathways for formation of antigen in penicillin allergy.

gen (Thiel et al., 1964). The very multiplicity of pathways which lead to formation of antigen makes it unlikely that a totally nonimmunogenic penicillin will be found. This does exclude the possibility of important quantitative differences among the penicillins in regard to immunogenicity, however.

The reason why formation of a stable bond with protein confers immunogenicity on a simple chemical is not clear. Some of the more important possibilities which come to mind are the following: combinations to protein could (1) retard destruction and excretion of the chemical, (2) facilitate its transfer into lymphoid cells, (3) permit its attachment to RNA, (4) aid in some other way its recognition as a potential antigen. The first two possibilities can probably be rejected on the basis of available evidence. An attrac- tive possibility would be that a combination of the chemical with an amino acid or peptide is required in order for attachment to RNA to take place. In the formation of antibody, binding of antigen to RNA may be required at some stage in the induction process.

Let us now consider the general problem of testing in drug allergy. Assume we have a patient receiving drug $A$ (Fig. 2) who has developed possible allergic symptoms. Now we will test him by injecting a small amount of $\mathrm{A}$ intradermally. A positive reaction will be an immediate skin reaction, that is a reaction of the wheal-and-erythema type which is read at 15-20 minutes. Let us further assume that in this particular patient, the antigenic determinant is a $\mathbf{B}^{\prime}$, derived from a breakdown product of A (Fig. 2). 
The first question we would like to ask is whether A or B can produce an allergic skin response or whether $B^{\prime}$ bound to skin protein must be formed. If $A$ and $B$ are structurally dissimilar, to $B^{\prime}$, allergic reactivity would not be expected. Even if A or B are structurally similar to $\mathbf{B}^{\prime}$, however, they will not produce an allergic response. They fail to do so because they have a single combining group, that is they are univalent (Farah, Kern and Eisen, 1960; Campbell and McCasland, 1944). In the precipitin reaction between antigen and antibody, the precipitating antigen must have multiple combining groups. This is necessary in order that an aggregate or lattice be formed with bivalent antibody. The univalent derivative by virtue of being able to combine with antibody but not precipitate with it is able to inhibit precipitation. Similar considerations apply to immediate allergic responses. In order for an antigen to elicit allergic manifestations it must have multiple combining groups. Thus $\mathbf{B}^{\prime}$ attached at multiple sites on skin protein molecules, will be able to produce an allergic response. A and $B$, to the extent to which they are structurally similar to $\mathbf{B}^{\prime}$ will compete for antibody and tend to inhibit the hypersensitivity response. Considered in this light, the failure of most drugs to produce immediate allergic responses is readily. explicable, for $B^{\prime}$ is formed at an insufficient rate to overcome the inhibitory effects of A and B. Drugs such as penicillin, which sometimes produce immediate skin responses, presumably do so because they have an unsual degree of protein reactivity.

How then can we overcome this problem of testing? We can prepare an effective test antigen if we tentatively identify $B^{\prime}$ and couple it with a protein or some other carrier in vitro forming a multivalent derivative. In order to evaluate the importance of the penicilloyl group in human penicillin allergy, we could use as our test material a penicilloylprotein conjugate. Protein conjugates of this nature have been used extensively in the experimental animal in evaluating hypersensitivity responses to simple chemical determinants. However, penicilloyl-proteins are potent inducers of antibody formation. Any large scale study in man would require the use of large numbers of normal subjects as well as those with penicillin allergy. If we employed penicilloyl proteins for testing, this would entail a substantial risk of causing penicillin allergy with our testing material.

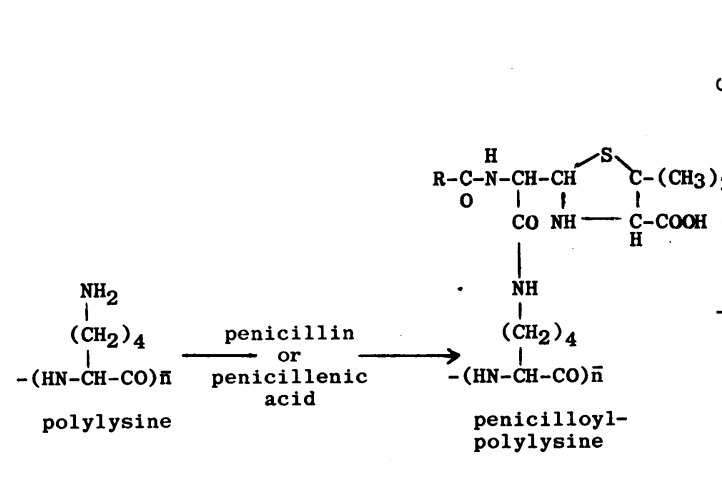

FIG. 4.-Formation of penicilloyl-polylysine.

Therefore, one would prefer to use a substio tute for protein as carrier for the penicilloy $\bar{B}$. group.

Since penicilloyl is attached to protein byes means of protein lysyl residues (Fig. 3), on€ can make a penicilloyl conjugate by using $\mathrm{a}$ polymer of lysine itself (Parker et al. $\vec{\nexists}$ 1962). Polylysine is a series of lysyl residues coupled together in peptide linkage. The $\varepsilon$-amino group is available for coupling to penicillin or penicillenic acid forming की product termed penicilloyl-polylysine (Fig. 4) $\stackrel{\oplus}{3}$ There is not sufficient space to review in? detail the data on immunogenicity of penioge loyl-polylysine. I think one would be justi-e fied in stating, however, that highly substituted penicilloyl-polylysine can be used in man witho very little if any risk of inducing antibodys formation (Parker, 1963; Rytel, Klion, Arlander and Miller, 1963). Polymers prepared@ using D-lysine, the unnatural configuration of the amino acid, are probably particularly safe in this regard (Parker and Thiel, 1963; Parker, 1964b).

We will now consider the results of skinö testing with penicillin-polylysine and other derivatives in man. Again it may be noted that the type of skin reaction we are concernedo with is an immediate skin reaction. What are the results if we test a group of subjects withô penicillin allergy? The results vary markedly

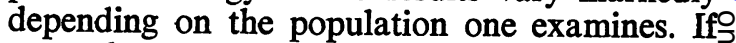
we take a random group of hospitalized patients with a history of penicillin allergy on average 5-10 years previously, the incidence of allergic reactions to penicilloyl-polylysine is of the order of $25-35 \%$ If we take a group of patients with recent $\omega$ penicillin reactions, well documented by a physician's observations during allergice symptoms, the incidence of positive reactions is much higher, of the order of $75-80 \%$ (Parker, 1963); in one recent study, positive 
skin reactions to penicilloyl-polylysine were obtained in $90 \%$ of subjects tested $2-2 \frac{1}{2}$ months after allergic symptoms had subsided. Penicillin and penicillin derivatives other than penicilloyl gave. positive skin responses in a minority of these subjects (Budd, Parker and Norden, 1964). If the patient is tested during or shortly after allergic symptoms, the incidence of positive skin responses to penicilloyl-polylysine is not as high.

The final question is whether penicilloylpolylysine can be used to predict penicillin reactions in subjects with no history of penicillin allergy. It should be emphasized strongly that this is not a question which can be evaluated by testing after the allergic response has taken place. A subject who was originally skin test negative to penicilloyl-polylysine may be skin test positive after the appearance of allergic symptoms. Moreover, a subject who has a strongly positive skin test may convert to a negative reaction after receiving penicillin. The skin test may remain negative during and after the development of allergic symptoms.

The results of prospective studies with penicilloyl-polylysine indicate that many potential penicillin reactions can be detected with this material. The patient with no history of penicillin allergy has a markedly increased risk of a penicillin reaction if he is skin test positive (Parker et al., 1962; Parker, 1963; Rytel et al., 1963). The increased risk is both for immediate and serum sickness type reactions. At one clinic, the incidence of penicillin reactions has been reduced to about $0.3 \%$ (or about 15 fold or more) by screening with penicilloyl-polylysine as well as a careful history (Summar, personal communication).

It is not established that all immediate systemic reactions to penicillin would be predicted by penicilloyl-polylysine. In view of the multiplicity of antigenic determinants in penicillin allergy it would be surprising if this were the case. I know of at least one instance where a patient who was skin negative to penicilloylpolylysine before receiving penicillin developed an immediate urticarial reaction after the drug was given. Despite these reservations penicilloyl-polylysine appears of considerable value both in the evaluation of suspected penicillin hypersensitivity and the prevention of serious allergic reactions.
It seems virtually certain that a similar approach to that taken experimentally in penicillin allergy (Parker et al., 1962; Levine and Ovary, 1961; Levine, 1960; De Weck and Eisen, 1960) would be productive in the study of hypersensitivity to other drugs. The major problem at present is the identification of the antigenic determinants involved. Ultimately we may reach the point where major antigens in the more important drug allergies are identified and appropriate diagnostic procedures available. Until that point is reached it will continue to be necessary to employ an empirical approach to most drug allergies (Parker, 1964a).

\section{REFERENCES}

Budd, M. A., Parker, C. W., and Norden, C. W. (1964): J. Amer. med. Ass. (in press).

Campbell, D. H., and McCasland, G. E. (1944): J. Immunol., 49, 315.

DeWeCK, A. L. (1962): Int. Arch. Allergy, 21, 20.

DEWECK, A. L., and EISEN, H. N. (1960): J. exp. Med., 112, 1227.

EISEN, H. N. (1959): Hypersensitivity to Simple Chemicals. In Lawrence, H. S., Cellular and Humoral Aspects of the Hypersensitive States. New York: P. B. Hoeber.

FARAH, F. S., KeRN, M., and EISEN, H. N. (1960): J. exp. Med., 112, 1211. HaRriS, J., and VAUGHN, J. M. (1961): J. Allergy,
32, 119.

LANDSTEINER, K. (1945): The Specificity of Serological Reactions. Cambridge: Harvard Univ. Press, (Rev. ed.).

LeviNe, B. B., and OVARY, Z. (1961): J. exp. Med., 114,875 .

LEviNE, B. B. (1960): J. exp. Med., 112, 1131.

LeY, A. B., Harris, J. P., BRINKLeY, M., Liles, B., JACK, J. A., and CAHAN, A. (1958): Science, 127, 1118 .

PARKER, C. W. (1964a): Chapter on drug allergy in the Clinical Section of Immunologic Diseases. Boston: Little, Brown (in press).

PARKER, C. W. (1964b): J. Immunology (in press)

PARKER, C. W., SHAPIRo, J., KeRN, M., and EISEN, H. N. (1962): J. exp. Med., 115, 821.

Parker, C. W. (1963): Amer. J. Med., 34, 747.

PARKer, C. W., and ThIEL, J. A. (1963): J. Lab. clin. Med., 62, 998. (Abstract).

Reisman, R. E., Rose, N. R., Witebsky, E., and Arbesman, C. E. (1962): J. Allergy, 33, 178.

Rytel, M., Klion, F. M., Arlander, T. R., and Miller, L. F. (1963):'J. Amer. med. Ass., 186, 894.

Sherman, W. B. (1947): Amer. J. Med., 3, 586.

Thiel, J. A., Mitchell, S., and Parker, C. W. (1964): J. Allergy, 35, 399.

VAN ARSDEL, P. P., Jr., Tobe, A. D., and PaSNick, L. J. (1963): J. Allergy, 34, 526 . 